\title{
126. Clinical Studies on the Variation in the Cerebral-Ventricul Temperature after Intracranial Operation
}

\author{
Tetsuya Shirao, Hitoshi Ichiki, Kazuo UchiYama, Yoshiaki OKuma \\ Kennosuke Takezako and Akinori NaKamura \\ 1st Department of Surgery, School of Medicine, Kagoshima University
}

The variations of ventricular temperature have benn reported prelin nary at the 20th Annual Meeting of the Japan Neurosurgical Societ Niigata City, on June 1st 1961.

The ventricular temperature in 48 cases were measured with thermist for 6 days postoperatively. The difference between the ventricular ter perature and the rectal tempature and its course were investigated. The temperature changings were analysed in relation to operations metho anesthesia method and postoperative cares. As remarkable postoperati changing in cerebro-spinal fluid temperature, bleeding and increasing protain and glucose were observed in some of Type 2 or Type 3.

According to the results described above, the measurement and its analy: of ventricular temperature might be a good indicator of diagnosis and pro nosis in central hyperthermia after intracranial operation.

\section{Ischemic Necrosis of the Skull Following Trephination}

\author{
Report of a Case \\ Yoshiaki Sugryama \\ Division of Neurosurgery, The Toyama Prefectural Central Hospital \\ Kozo YaJIMA and Komei UEKI \\ Department of Neurosurgery, Brain Research Institute, Niigata University \\ School of Medicine
}

Epidural or subdural hematoma is not rarely encountered after ver tricular puncture in patients with obstructiue hydrocephalus or congenit: hydrocephalus. But there has been no report on lenticularly shaped sku distension developed adjacent to a burr hole assuming radiologically, chr nic ossifying hematoma. The patient was 11-year-old boy with convulsi seizure. Five years previously he gradually developed nausea, vomiting an 